\title{
ROTARU ALPHA - CONVEX FUNCTIONS
}

\author{
SUBHAS S. BHOOSNURMATH AND S. R. SWAMY
}

\begin{abstract}
Let $S^{*}(a, b)$ denote the class of analytic functions $f$ in the unit disc $E$, with $f(0)=f^{\prime}(0)-1=0$, satisfying the condition $\left|\left(z f^{\prime}(z) / f(z)\right)-a\right|<b, a \in C,|a-1|<b \leq \operatorname{Re}(a), z \in E$. In this paper the class $S^{*}(\alpha, a, b)$ of functions $f$ analytic in $E$, with $f(0)=$ $f^{\prime}(0)-1=0, f(z) f^{\prime}(z) / z \neq 0$ for $z$ in $E$ and satisfying in $E$ the condition $|J(\alpha, f)-a|<b, a \in C,|a-1|<b \leq \operatorname{Re}(a)$, where $J(\alpha, f)=(1-$ $\alpha)\left(z f^{\prime}(z) / f(z)\right)+\alpha\left(\left(z f^{\prime}(z)\right)^{\prime} / f^{\prime}(z)\right), \alpha$ a non-negative real number is introduced. It is proved that $S^{*}(\alpha, a, b) \subset S^{*}(a, b)$, if $\alpha>(4 b / c)|\operatorname{Im}(a)|$, $c=\left(b^{2}-|a-1|^{2}\right) / b$. Further a representation formula for $f \in S^{*}(\alpha, a, b)$ and an inequality relating the coefficients of functions in $S^{*}(\alpha, a, b)$ are obtained.
\end{abstract}

\section{Introduction}

Let $V$ denote the class of functions $\int$ analytic in the unit disc $E$, with $f(0)=$ $f^{\prime}(0)-1=0$. A function $f$ of $V$ is said to belong to $S^{*}(\rho)$, the class of starlike function of order $\rho$, if $\operatorname{Re}\left(z f^{\prime}(z) / f(z)\right)>\rho, 0 \leq \rho<1$. The class $S^{*}$ of starlike functions is identified by $S^{*}(0) \equiv S^{*}$. In [6] Rotaru investigated properties of the class $S^{*}(a, b)$ of functions $f \in V$ satisfying $\left|\left(z f^{\prime}(z) / f(z)\right)-a\right|<b, z \in E$, where $a \in C,|a-1|<b \leq \operatorname{Re}(a)$. It is clear that $S^{*}(a, b) \subset S^{*}(\operatorname{Re}(a)-b) \subset S^{*}$. Let $K(a, b)$ denote the class of functions $f$ in $V$ for which $z f^{\prime} \in S^{*}(a, b)$.

In this paper we combine the notions of Rotaru starlike functions [6] and alpha-convex functions [2] to obtain a new subclass of starlike functions.

Received September 30, 1991.

AMS (MOS) subject classification (1980):30C45.

Keywords: Univalent, Starlike and Alpha-Convex Functions. 
We now introduce the class $S^{*}(\alpha, a, b)$ of functions $f \in V$ with $f(z) f^{\prime}(z) / z \neq$ $0, z \in E$, satisfying in $E$ the condition

$$
|J(\alpha, f)-a|<b, \quad a \in C, \quad|a-1|<b \leq \operatorname{Re}(a),
$$

where $J(\alpha, f)=(1-\alpha)\left(z f^{\prime}(z) / f(z)\right)+\alpha\left(z f^{\prime}(z)\right)^{\prime} / f^{\prime}(z)$ and $\alpha$ a non-negative real number. Functions in $S^{*}(\alpha, a, b)$ are called Rotaru alpha-convex functions.

Note that $S^{*}(0, a, b) \equiv S^{*}(a, b)$ and we investigate a few properties of the class $S^{*}(\alpha, a, b)$.

2. We require the following lemmas to prove our main theorem

Lemma 2.1. Let $a \in C,|a-1|<b \leq \operatorname{Re}(a)$ and $p(z)$ be analytic in $E$ with $p(0)=1$. Then $|p(z)-a|<b, z \in E$, if and only if, there exists a function $w$ analytic in $E$ satisfying $w(0)=0,|w(z)|<1$ for $z \in E$ such that

$$
p(z)=\frac{1+A w(z)}{1+\bar{B} w(z)}, \quad z \in E
$$

where $A=\left(b^{2}-|a|^{2}+a\right) / b$ and $B=(1-a) / b$.

The proof of this lemma follows by an application of Schwarz's lemma as in Rotaru [6].

From now on $A$ and $B$ will be as in lemma 2.1 and also $c=\left(b^{2}-|a-1|^{2}\right) / b$.

Next we have the well known Jack's lemma [1].

Lemma 2.2. Let $w(z)$ be regular in $E$ with $w(0)=0$. If there exists a $z_{0} \in E$ such that $\max _{|z| \leq\left|z_{0}\right|}|w(z)|=\left|w\left(z_{0}\right)\right|$ then $z_{0} w^{\prime}\left(z_{0}\right)=k w\left(z_{0}\right)$ for some $k \geq 1$.

It is well known that all $\alpha$-convex functions are starlike [2]. We now prove an analogous theorem for the class $S^{*}(\alpha, a, b)$.

Theorem 2.1. Let $f(z) \in S^{*}(\alpha, a, b), \alpha>(4 / c)|\operatorname{Im}(a)|$. Then $f(z) \in$ $S^{*}(a, b)$. 
Proof. Define an analytic function $w(z)$ in $E$ by

$$
\frac{z f^{\prime}(z)}{f(z)}=\frac{1+A w(z)}{1+\bar{B} w(z)}
$$

Clearly $w(0)=0$ and $w(z) \neq-1 / \bar{B}$ in $E$. In view of lemma 2.1, it sufficies to show that $|w(z)|<1$. Suppose there exists a $z_{0} \in E$ such that $\max _{|z| \leq\left|z_{0}\right|}|w(z)|=$ $\left|w\left(z_{0}\right)\right|=1$, then by lemma 2.2 ,

$$
z_{0} w^{\prime}\left(z_{0}\right)=k w\left(z_{0}\right), \quad k \geq 1
$$

From (2.1) and (2.2) we get

$$
\left|J\left(\alpha, f\left(z_{0}\right)\right)-a\right|=\iota\left|\frac{B+(1+A B+(\alpha c k) / b) w\left(z_{0}\right)+A w^{2}\left(z_{0}\right)}{1+(A+\bar{B}) w\left(z_{0}\right)+A \bar{B} w^{2}\left(z_{0}\right)}\right|
$$

where $c=\left(b^{2}-|a-1|^{2}\right) / b>0$. Now $\left|J\left(\alpha, f\left(z_{0}\right)\right)-a\right|>b$, provided

$$
\left|B+(1+A B+(\alpha c k) / b) w\left(z_{0}\right)+A w^{2}\left(z_{0}\right)\right|^{2}>\left|1+(A+\bar{B}) w\left(z_{0}\right)+A \bar{B} w^{2}\left(z_{0}\right)\right|^{2} .
$$

Condition (2.3) reduces to the following:

$$
(\alpha c k) / 2 b+1+\operatorname{Re}(A B)+\operatorname{Re}\left[(A+\bar{B}) w\left(z_{0}\right)\right]>0
$$

or equivalently

$$
\begin{aligned}
\frac{\alpha c(k-1)}{2 b}+1+\operatorname{Re}(A) \operatorname{Re}(B) & -\operatorname{Im}(A) \operatorname{Im}(B)+\operatorname{Re}(A+\bar{B}) \operatorname{Re}\left(w\left(z_{0}\right)\right) \\
& -\operatorname{Im}(A+\bar{B}) \operatorname{Im}\left(w\left(z_{0}\right)\right)+\frac{\alpha c}{2 b}>0
\end{aligned}
$$

Since $\frac{\alpha c}{2 b}(k-1) \geq 0$ and $\operatorname{Im}(A) \operatorname{Im}(B)<0,(2.4)$ holds provided $\frac{\alpha c}{2 b} \pm \operatorname{Im}(A+$ $\bar{B})>0$ and $1+\operatorname{Re}(A) \operatorname{Re}(B) \pm \operatorname{Re}(A+\overline{I B})>0$. Now, since $\alpha>(4 / c)|\operatorname{Im}(a)|$ we have

$$
\frac{\alpha c}{2 b} \pm \operatorname{Im}(A+\bar{B})>0
$$

and

$$
\begin{array}{r}
1+\operatorname{Re}(A) \operatorname{Re}(B) \pm \operatorname{Re}(A+\bar{B})=(1 \pm \operatorname{Re}(A))(1 \pm \operatorname{Re}(B)) \geq 0 \\
\text { since }|A| \leq 1 \text { and }|B|<1
\end{array}
$$


This means that $f\left(z_{0}\right) \in S^{*}(\alpha, a, b)$, a contradiction. Thus the proof is complete.

Corollary 2.1. Let $f(z) \in S^{*}(\alpha, a, b), \alpha>0$. Then $f(z) \in S^{*}(a, b)$.

The above corollary follows by taking $a=\bar{a}$ in theorem 2.1 .

Corollary 2.2. Let $\alpha>0, b>1 / 2$ and $f(z) \in S^{*}(\alpha, b, b)$. Then $f(z) \in$ $S^{*}(b, b)$.

The above result is obtained if, in corollary 2.1 , we take $a=b, b>1 / 2$.

Remark. Corollary 2.2 improves a result of Miller, Mocanu and Reade [4] who proved it when $b \geq 1$.

Let us choose $a=(1+\rho-2 \rho \beta) / 2(1-\beta)$ and $b=(1-\rho) / 2(1-\beta)$, where $0 \leq \rho<1$ and $0<\beta \leq 1$. Then the undermentioned corollary follows now from corollary 2.1 .

Corollary 2.3. Let $\alpha>0,0 \leq \rho<1,0<\beta \leq 1$ and $f \in V$ with $f(z) f^{\prime}(z) / z \neq 0$ for $z$ in $E$. Then

$$
\left|\frac{\left(z f^{\prime}(z) / f(z)\right)-1}{2 \beta\left(\left(z f^{\prime}(z) / f(z)\right)-\rho\right)-\left(\left(z f^{\prime}(z) / f(z)\right)-1\right)}\right|<1, \quad \text { for } z \text { in } E .
$$

whenever

$$
\left|\frac{J(\alpha, f(z))-1}{2 \beta(J(\alpha, f(z))-\rho)-(J(\alpha, f(z))-1}\right|<1, \quad \text { for } z \text { in } E
$$

where $J(\alpha, f(z))=(1-\alpha)\left(z f^{\prime}(z) / f(z)\right)+\alpha\left(z f^{\prime}(z)\right)^{\prime} / f^{\prime}(z)$ and $\alpha$ a non-negative real number.

Remark. For $\rho=(1-\lambda) /(1+\lambda)$ and $\beta=(1+\lambda) / 2,0<\lambda \leq 1$, corollary 2.3 agrees with theorem 1 of Padmanabhan and Bharati [5].

Theorem 2.2. For $(4 / c)|\operatorname{Im}(a)|<\beta<\alpha, S^{*}(\alpha, a, b) \subset S^{*}(\beta, a, b)$.

Proof. Let $f(z) \in S^{*}(\alpha, a, b)$. Form theorem 2.1 and the identity

$$
\left.J(\beta, f(z))-a=\frac{\beta}{\alpha}[J(\alpha, f(z))-a]+\left(1-\frac{\beta}{\alpha}\right)\left[z f^{\prime}(z) / f(z)\right)-a\right], \quad \beta<\alpha,
$$


we arrive at the desired result $f(z) \in S^{*}(\beta, a, b)$.

Corollary 2.4. For $\alpha \geq 1>(4 / c)|\operatorname{Im}(a)|, S^{*}(\alpha, a, b) \subset K(a, b)$.

3. In this section, we obtain an important integral representation for the elements of $S^{*}(\alpha, a, b)$.

Theorem 3.1. Let $f(z) \in S^{*}(\alpha, a, b), \alpha>(4 / c)|\operatorname{Im}(a)|$, and if for $(4 / c)$ $|\operatorname{Im}(a)|<\beta<\alpha$ we choose the branch of $\left[z f^{\prime}(z) / f(z)\right]^{\beta}$ which is equal to 1 when $z=0$, then the function $F_{\beta}(z)=f(z)\left[z f^{\prime}(z) / f(z)\right]^{\beta}$ is in $S^{*}(a, b)$.

Proof. A simple calculation yields

$$
\frac{z F_{\beta}^{\prime}(z)}{F_{\beta}(z)}=J(\beta, f(z))
$$

Since $f(z) \in S^{*}(\alpha, a, b)$, by theorem 2.2 , we have

$$
\left|\frac{z F_{\beta}^{\prime}(z)}{F_{\beta}(z)}-a\right|=|J(\beta, f(z))-a|<b,
$$

for $(4 / c)|\operatorname{Im}(a)|<\beta<\alpha$. IIence $F_{\beta}(z) \in S^{*}(a, b)$.

Now we consider the converse problem. Given the function $F(z) \in S^{*}(a, b)$ and $\alpha>(4 / c)|\operatorname{Im}(a)|$ is the solution

$$
f(z)=\left\{\frac{1}{\alpha} \int_{0}^{z} F^{1 / \alpha}(t) t^{-1} d t\right\}^{\alpha}
$$

of the differential equation

$$
F(z)=f(z)\left[z f^{\prime}(z) / f(z)\right]^{\alpha},
$$

with boundary condition $f(0)=0$, a function in $S^{*}(\alpha, a, b)$ ? The answer is yes, and our solution provides us with an integral representation formula for functions is $S^{*}(\alpha, a, b)$.

Theorem 3.2. Let $F(z) \in S^{*}(a, b)$ and $\alpha>(4 / c)|\operatorname{Im}(a)|$. Then $f(z)$ defined by (3.1) belongs to $S^{*}(\alpha, a, b)$. 
The proof of this theorem consists of showing that $f(z)$ is well defined, regular in $E$ and is in $S^{*}(\alpha, a, b)$. The technique is similar to the one employed in $[3$, Theorem 5$]$ and is omitted.

4. In this section we obtain an inequality for the coefficients of functions in $S^{*}(\alpha, a, b)$

Theorem 4.1. Let $f(z) \in S^{*}(\alpha, a, b)$ and let $s_{1}=0, t_{1}=1-(A / \bar{B})$, $s_{m}=(1-\alpha)\left(\beta_{m}-\alpha_{m}\right)+\alpha \gamma_{m-1}, t_{m}=(\alpha-(A / \bar{B})) \alpha_{m}+(1-\alpha) \beta_{m}+\alpha \gamma_{m-1}$, $m=2,3,4, \ldots$, where $\alpha_{m}, \beta_{m}$ and $\gamma_{m}$ are defined by

$$
\begin{aligned}
& \alpha_{m}=\sum_{k=1}^{m}(m-k+1) a_{k} a_{m-k+1}, \\
& \beta_{m}=\sum_{k=1}^{m} k(m-k+1) a_{k} a_{m-k+1}, \\
& \gamma_{m}=\sum_{k=1}^{m} k(k+1) a_{k+1} a_{m-k+1} .
\end{aligned}
$$

Then the coefficients $a_{n}$ satisfy the following inequality:

$$
\sum_{m=1}^{n}\left|s_{m}\right|^{2} \leq|B|^{2} \sum_{m=1}^{n}\left|t_{m}\right|^{2}, \quad n=2,3,4, \ldots
$$

Proof. Since $f(z) \in S^{*}(\alpha, a, b)$,

$$
(1-\alpha) \frac{z \int^{\prime}(z)}{f(z)}+\alpha\left[\frac{\left(z \int^{\prime}(z)\right)^{\prime}}{f^{\prime}(z)}\right]=\frac{1+A w(z)}{1+\bar{B} w(z)},
$$

where $w$ is analytic in $E, w(0)=0$ and $|w(z)|<1$ in $E$. This gives

$$
\begin{aligned}
& (1-\alpha)\left[z\left(f^{\prime}(z)\right)^{2}-\int(z) f^{\prime}(z)\right]+\alpha z f(z) f^{\prime \prime}(z) \\
= & \left.-\bar{B} w(z)\left[\alpha-\frac{A}{\bar{B}}\right) f(z) f^{\prime}(z)+(1-\alpha) z\left(f^{\prime}(z)\right)^{2}+\alpha z f(z) f^{\prime \prime}(z)\right] .
\end{aligned}
$$

Given $f(z)=z+\sum_{n=2}^{\infty} a_{n} z^{n}$, we note that

$$
f(z) f^{\prime}(z)=\sum_{m=1}^{\infty} \alpha_{m} z^{m},\left(f^{\prime}(z)\right)^{2}=\sum_{m=1}^{\infty} \beta_{m} z^{m-1}, f(z) f^{\prime \prime}(z)=\sum_{m=1}^{\infty} \gamma_{m} z^{m}
$$


where $\alpha_{m}, \beta_{m}$ and $\gamma_{m}$ are defined in (4.1). Thus (4.2) becomes

$$
\begin{aligned}
& (1-\alpha) \sum_{m=1}^{\infty}\left(\beta_{m}-\alpha_{m}\right) z^{m}+\alpha \sum_{m=1}^{\infty} \gamma_{m} z^{m+1} \\
= & -\bar{B} w(z)\left\{(1-\alpha) \sum_{m=1}^{\infty} \beta_{m} z^{m}+\left(\alpha-\frac{A}{\bar{B}}\right) \sum_{m=1}^{\infty} \alpha_{m} z^{m}+\alpha \sum_{m=1}^{\infty} \gamma_{m} z^{m+1}\right\}
\end{aligned}
$$

which simplifies to

$$
\sum_{m=1}^{\infty} s_{m} z^{m}=-\bar{B} w(z)\left\{\sum_{m=1}^{\infty} t_{m} z^{m}\right\}
$$

Now from (4.3) we get

$$
\left|\sum_{m=1}^{n} s_{m} z_{m}+\sum_{m=n+1}^{\infty} h_{m} z^{m}\right|<|B|\left|\sum_{m=1}^{n-1} \iota_{m} z^{m}\right|
$$

where $h_{m}$ 's are some complex numbers. This yields

or

$$
\sum_{m=1}^{n}\left|s_{m}\right|^{2}+\sum_{m=n+1}^{\infty}\left|h_{m}\right|^{2} \leq|B|^{2} \sum_{m=1}^{n-1}\left|t_{m}\right|^{2}
$$

$$
\sum_{m=1}^{n}\left|s_{m}\right|^{2} \leq|B|^{2} \sum_{m=1}^{n-1}\left|t_{m}\right|^{2}
$$

The above result is sharp for the function

$$
\begin{aligned}
f_{\alpha}(z) & =\left\{\frac{1}{\alpha} \int_{0}^{z} t^{1 / \alpha-1}\left(1+\frac{1-\bar{a}}{b} t\right)^{\frac{c}{\alpha(1-\bar{a})}} d t\right\}^{\alpha} \quad \text { if } \quad a \neq 1 \\
& =\left\{\frac{1}{\alpha} \int_{0}^{z} t^{1 / \alpha-1} e^{\frac{b t}{\alpha}} d t\right\}^{\alpha} \text { if } \quad \iota=1
\end{aligned}
$$

\section{References}

[1] I. S. Jack, "Functions starlike and convex of order $\alpha$ ", J. London Math. Soc., 2 (3) (1971), 469-479.

[2] S. S. Miller, P. T. Mocanu and M. O. Reade, "All $\alpha$-convex functions are starlike and univalent", Proc. Amer. Math. Soc., 37, 2 (1973), 553-554. 
[3] S. S. Miller, P. T. Mocanu and M. O. Reade, "Bazilevic functions and generalized convexity", Rev. Roum. D. Math. Pure. Et. Appli., 19 (1974), 213-224.

[4] S. S. Miller, P. T. Mocanu and M. O. Reade, "Janowski $\alpha$-convex functions", Annal. UMSL. Polon., 29 (1975), 93-98.

[5] K. S. Padmanabhan and R. Bharati, "On a subclass of univalent functions-I", Annal. Polon. Math., XLIII (1983), 57-64.

[6] P. Rotaru, "Subclasses of starlike functions", Mathematica, 29/52, 2 (1987), 183-191.

Department of Mathematics, Karnatak University, Dharwad-580 003, INDIA.

Department of Mathematics, J. M. Institute of Technology, Chitradurga-577 502, INDIA. 